\title{
Camptocormia: um relato de caso bizarro e a sua adequada referenciação
}

Jorge Hernâni-Eusébio, ${ }^{1}$ Ricardo Jorge Silva, ${ }^{2}$ Álvaro Machado ${ }^{3}$

\section{RESUMO}

Introdução: A camptocormia (flexão anterior da coluna toraco-lombar) é um diagnóstico secundário a causas neurológicas (particularmente à doença de Parkinson), causas ortopédicas e outras (intoxicação farmacológica e síndromas paraneoplásicos). Quando associada a doenças neurológicas, esta reverte integralmente se o utente assumir o decúbito dorsal, o que não se verifica quando a causa é ortopédica. Este achado puramente clínico é fulcral para a referenciação de um utente da forma mais correta e tomada de decisão sobre o plano a adotar.

Descrição do caso: Homem de 65 anos, sem antecedentes de relevo ou uso de medicação habitual. Observado em consulta de neurologia, entrando no consultório com marcada flexão anterior da coluna toraco-lombar (camptocormia). $O$ utente foi previamente orientado pelo médico de família para consulta de ortopedia por esta queixa, com imagiologia de achatamento em cunha da vértebra T12. Também acompanhado por medicina física e reabilitação que, após tratamento fisiátrico, o referencia à neurologia por suspeita de causa neurológica da camptocormia. Ao exame objetivo apresentava camptocormia marcada que não revertia no decúbito dorsal. Sem tremor, rigidez, discinésia ou outros sintomas extrapiramidais. Sem incontinência esfincteriana. Comentário: A referenciação de casos de camptocormia pode ser complexa, permitindo a abordagem holística do utente compreender a sua globalidade e a dos seus sintomas, ponderando os achados indicativos da causa primária do problema. Neste caso, a não reversão da camptocormia com o decúbito dorsal, a ausência de tremor/sintomas extrapiramidais e a documentação imagiológica de possível causa ortopédica tornam a referenciação à consulta de neurologia menos pertinente que a referenciação feita pelo médico de família para consulta de ortopedia. Podemos, assim, gerir de forma mais profícua a situação do utente $\mathrm{e}$ as suas expectativas quanto à resolução do problema.

Foi dado pelo utente o consentimento informado, livre e esclarecido para a recolha de dados clínicos e imagens fotográficas, assim como para publicação.

Palavras-chave: Curvaturas da coluna; Curvaturas da coluna/diagnóstico; Curvaturas da coluna/etiologia;Atrofia muscular/diagnóstico; Atrofia muscular/etiologia; Camptocormia.

\section{INTRODUÇÃO}

$\Lambda$ camptocormia (flexão anterior da coluna toraco-lombar) figura como um diagnóstico secundário a causas neurológicas, ortopédicas e outras (intoxicação farmacológica, síndromas paraneoplásicos, entre outros).

Quando é secundária a doenças neurológicas, esta reverte integralmente se o utente assumir o decúbito

1. Médico Interno em Medicina Geral e Familiar. USF do Minho, ACeS Cávado I Braga.

2. Médico Especialista em Medicina Geral e Familiar. USF do Minho, ACeS Cávado I - Braga.

3. Médico Especialista em Neurologia, Serviço de Neurologia, Hospital de Braga. dorsal. O mesmo não se verifica quando a causa é ortopédica. Este achado do exame objetivo é fundamental para que a orientação e eventual referenciação do utente seja feita da maneira mais adequada.

O caso clínico descrito permitirá revisitar as causas primárias de camptocormia e ainda a orientação destas, de forma a gerir mais proficuamente a situação do utente e as suas expectativas quanto à resolução do problema.

\section{DESCRIÇÃO DO CASO}

Utente do sexo masculino com 65 anos, observado em contexto de consulta hospitalar de neurologia. Vem 
acompanhado da esposa, com quem reside. É operário numa fábrica de candeeiros, encontrando-se no ativo. Tem o $4^{\circ}$ ano de escolaridade.

Tem, como antecedentes pessoais, hérnia inguinal à esquerda (intervencionada cirurgicamente há um ano) e fratura vertebral de T12 documentada há dois anos. Ex-fumador (10 unidades maço-ano). Antecedentes familiares irrelevantes. Não faz uso de medicação habitual e desconhece alergias alimentares ou medicamentosas.

Foi referenciado há três anos pelo médico de família (MF) para consulta hospitalar de ortopedia por hipercifose lombar em progressivo agravamento. Efetuou estudo imagiológico com tomografia computorizada (TC) e radiografia onde é descrito achatamento em cunha anterior do corpo da vértebra T12 em relação com discreta fratura por compressão, com sinais acentuados de espondilose e deformidade de todo o ráquis lombar e da metade inferior do ráquis torácico. Registada também cifose toraco-lombar com perda da habitual lordose lombar. Foi também realizado um estudo de possíveis causas reumatológicas, com HLA B27 e fator reumatoide negativos.

Na consulta de ortopedia é realizada ressonância magnética (RM), que demonstra achados sobreponíveis aos da TC. É, então, decidido o encaminhamento do utente para consulta de medicina física e reabilitação (MFR), onde realiza fisioterapia e hidrocinesioterapia, com escassa melhoria dos sintomas. Este outcome acabou por instaurar no utente algum descontentamento, já que tinha a expectativa de melhoria marcada do quadro. Queixa-se também das constantes e dispendiosas deslocações ao hospital para realização dos tratamentos com uma contrapartida pouco mensurável em termos de resultados.

É encaminhado, pela MFR, para consulta de neurologia por apresentar, segundo o descrito, "movimentos involuntários dos dedos durante a marcha sem balanceio dos membros superiores", colocando-se a hipótese da existência de uma causa neurológica para a camptocormia do utente.

À entrada, no consultório médico, já em contexto da consulta de neurologia observada, apresenta marcha com flexão marcada do tronco para a frente (camptocormia), sem observação de sinais extrapiramidais à primeira vista (Figuras 1 e 2). Observa-se também que

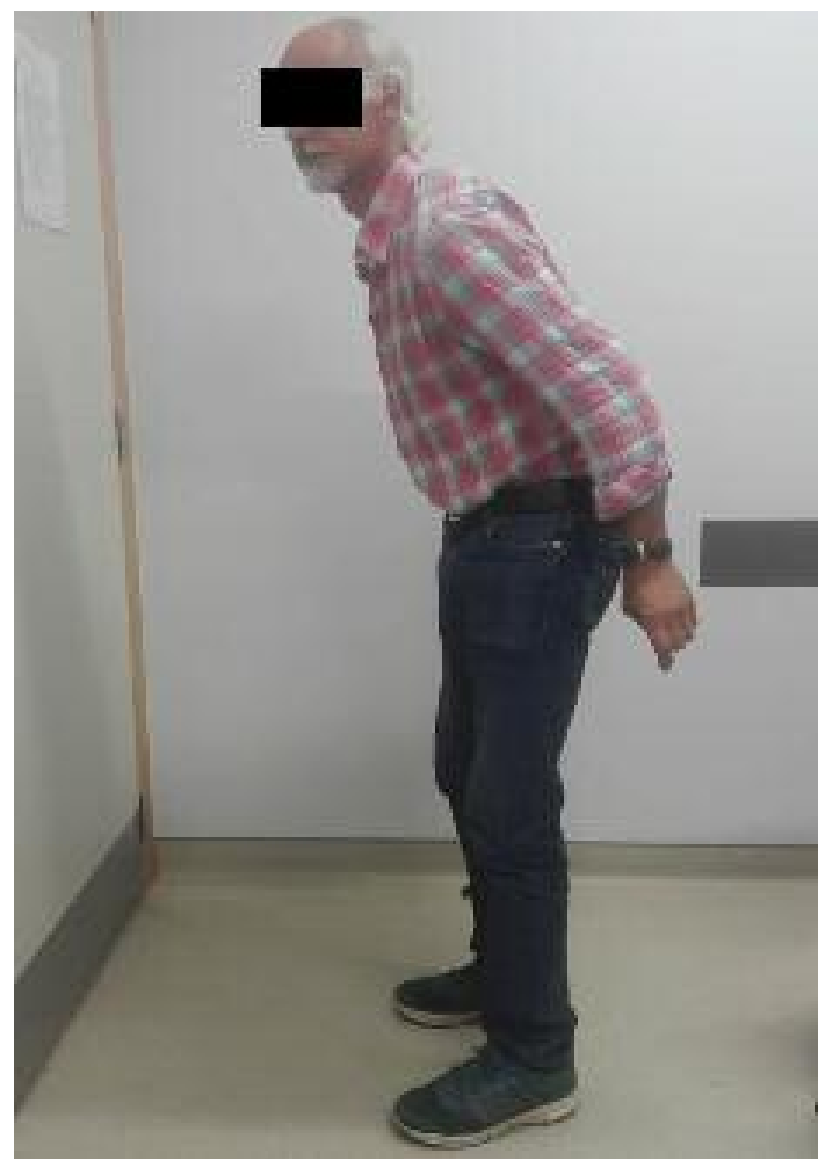

Figura 1. Camptocormia em ortostatismo.

a camptocormia esbate apenas ligeiramente na posição sentada (Figura 3).

Conta o utente: "não consigo endireitar as costas vai para três anos a esta parte" (sic). Queixa-se também de lombalgia moderada com dois anos de evolução, de ritmo mecânico, com agravamento ao final do dia, a agravar desde há meio ano. Esta não tem fatores de alívio e agrava com o esforço físico realizado no trabalho. Nega irradiação da lombalgia, possíveis alterações esfincterianas ou parestesias, colocando no topo da sua lista de preocupações a incapacidade para "endireitar a coluna" (sic).

Quando questionado sobre a eficácia dos tratamentos oferecidos até então, refere sentir-se frustrado porque "a medicação, a fisioterapia e a piscina não me tiram as dores nem me colocam as costas como deve ser" (sic), existindo registos de MFR que corroboram a não melhoria do quadro com os tratamentos de fisioterapia e hidrocinesioterapia. 


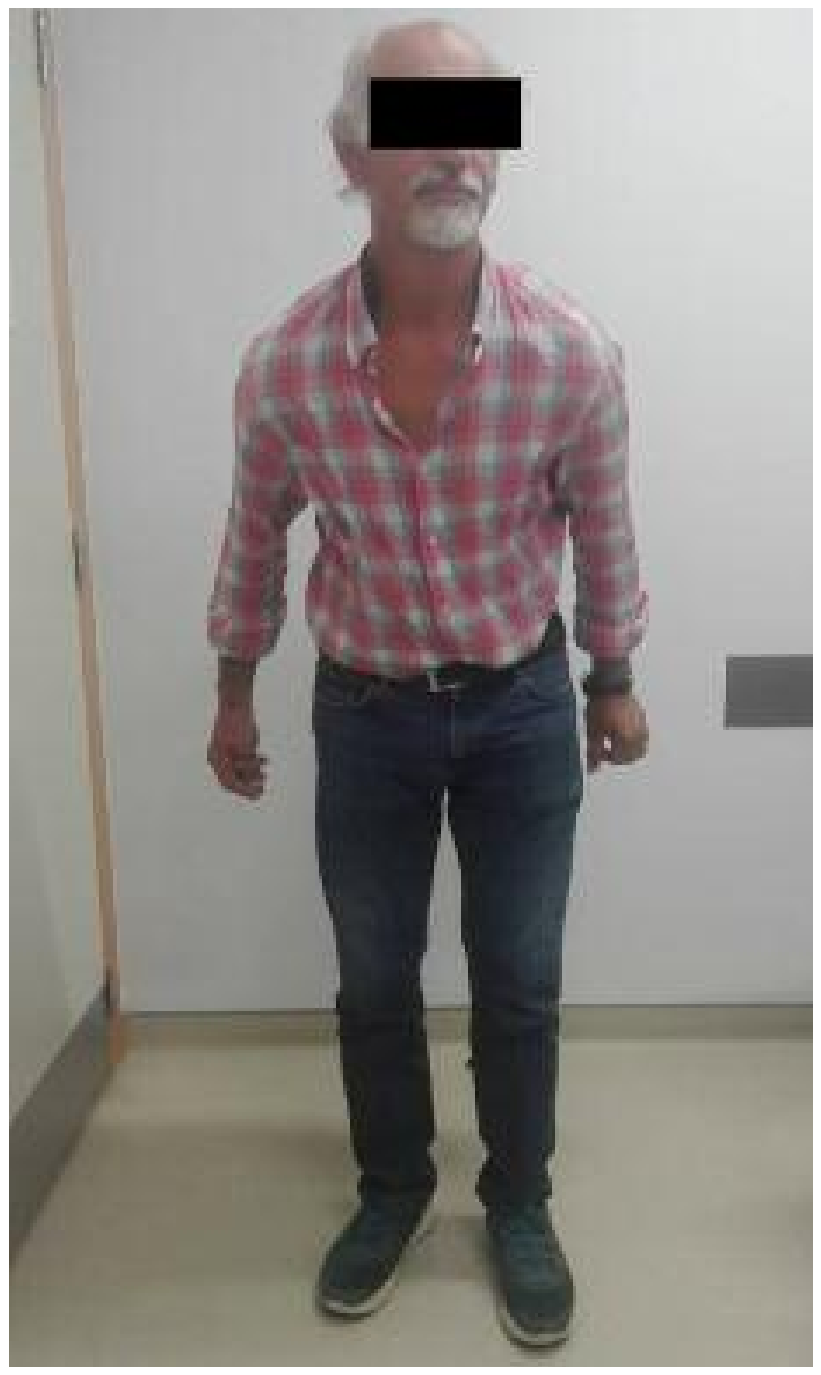

Figura 2. Camptocormia em ortostatismo.

Quanto a achados positivos relevantes ao exame objetivo, destaque-se a camptocormia marcada com limitação da extensão da coluna toraco-lombar, sendo que esta reduz lentamente com a posição de decúbito dorsal, não esbatendo, porém, por completo (Figuras 4 e 5). Negam-se sinais extrapiramidais, rigidez, tremor essencial ou outros sinais que possam indicar alguma causa primária de foro neurológico para a camptocormia encontrada.

Deste modo, e tendo em conta a mais que provável génese ortopédica da camptocormia, decide-se pelo reencaminhamento do utente para as consultas de ortopedia e MFR (totalidade das referenciações entre con-

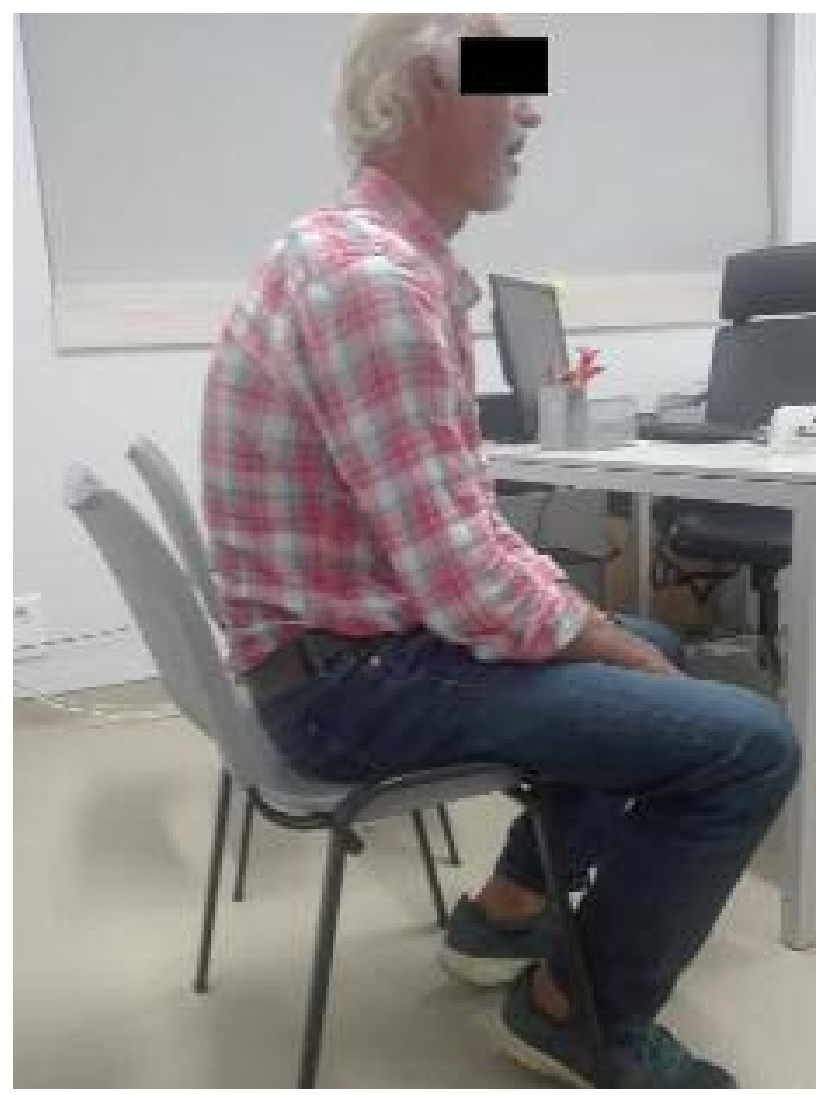

Figura 3. Ligeira diminuição da camptocormia com a posição sentada.

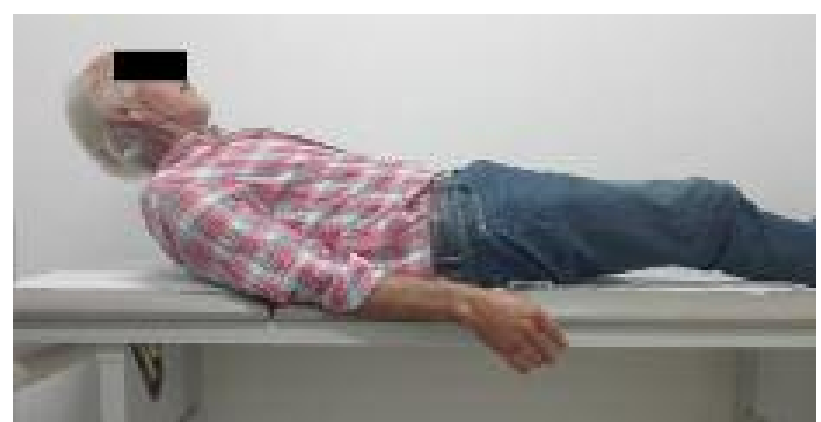

Figura 4. Camptocormia não reversível com o decúbito dorsal.

sultas em cronograma na Figura 6), sendo explicada ao utente a etiologia do seu problema e a orientação a dar ao mesmo.

\section{COMENTÁRIO}

O diagnóstico de camptocormia é, per se, bizarro e interessante. Se se lhe juntar a complexidade do racio- 


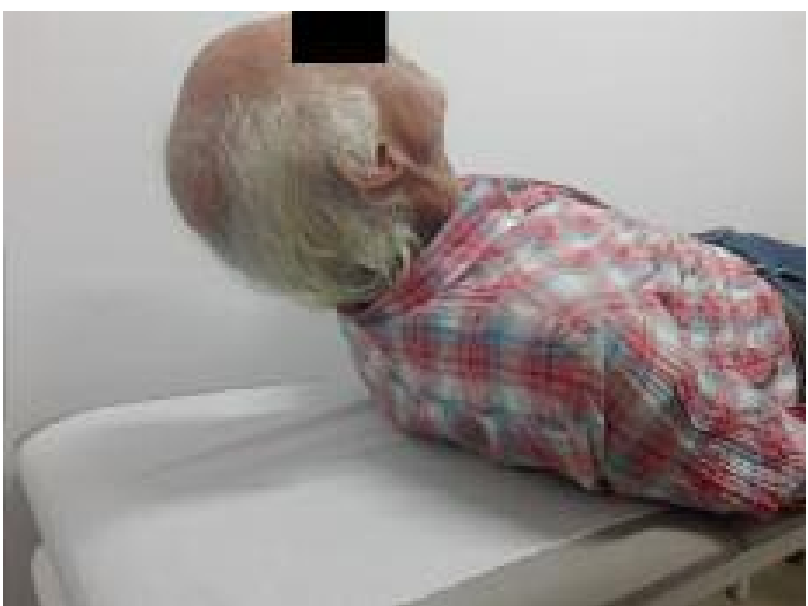

Figura 5. Camptocormia não reversível com o decúbito dorsal.

cínio por detrás da sua orientação, verifica-se que a referenciação dos utentes com camptocormia pode ser muito complexa.

Assim, espera-se que este relato de caso possa servir de exemplo à conduta na presença de futuros diagnós- ticos de camptocormia tanto nos CSP como nos cuidados de saúde secundários.

Logo, e numa lógica de raciocínio diagnóstico encadeado, a camptocormia (também conhecida como bent spine syndrome ou cyphose histérique $)^{1}$ pode ser secundária às causas de camptocormia vigentes na Tabela $2{ }^{2}$ figurando as doenças do sistema nervoso central (principalmente a doença de Parkinson) como as mais frequentes, seguidas das causas ortopédicas. Mais raramente, a camptocormia pode ser secundária à toxicidade por fármacos (como levodopa, antipsicóticos, valproato de sódio, entre outros), a síndromas paraneoplásicos ou a contextos psiquiátricos de muito difícil diagnóstico. ${ }^{2}$

Deve-se ainda diferenciar cuidadosa e corretamente a camptocormia da Síndroma de Torre de Pisa (cursando esta com flexão tónica lateral do tronco e da cabeça, normalmente associada a fenómenos escolióticos). ${ }^{2}$

Reitere-se que a literatura afirma que quase todos os utentes com camptocormia têm espondiloartrose, sendo esta um fator de risco per se para desenvolver camp-

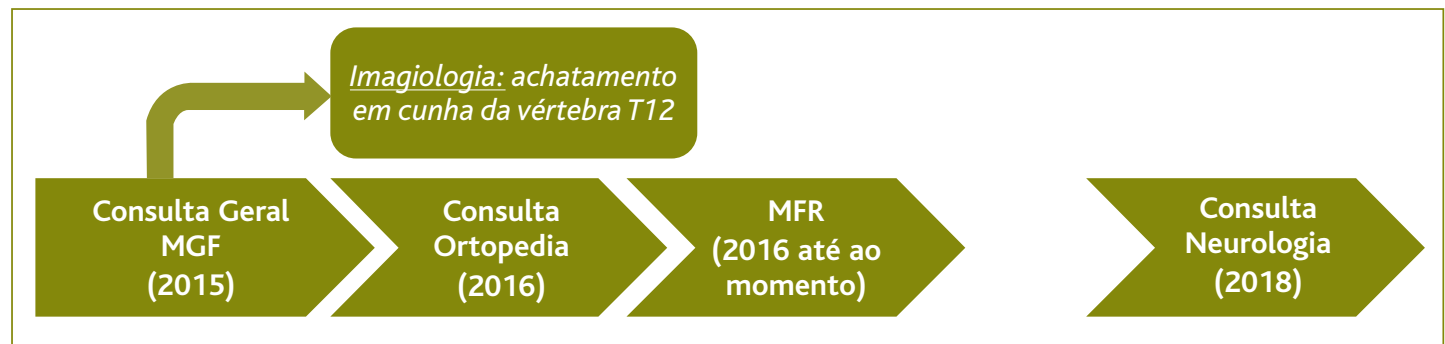

Figura 6. Cronograma de seguimento do utente desde 2015.

TABELA 1. Causas primárias de camptocormia
\begin{tabular}{l|l} 
Causas neurológicas & $\begin{array}{l}\text { Doença de Parkinson } \\
\text { Distrofias musculares } \\
\text { Esclerose lateral amiotrófica }\end{array}$ \\
\hline Causas ortopédicas & $\begin{array}{l}\text { Hérnia discal } \\
\text { Fratura vertebral } \\
\text { Trauma }\end{array}$ \\
\hline Outras causas & $\begin{array}{l}\text { Fármacos } \\
\text { Síndromas paraneoplásicos } \\
\text { Causas psiquiátricas }\end{array}$
\end{tabular}

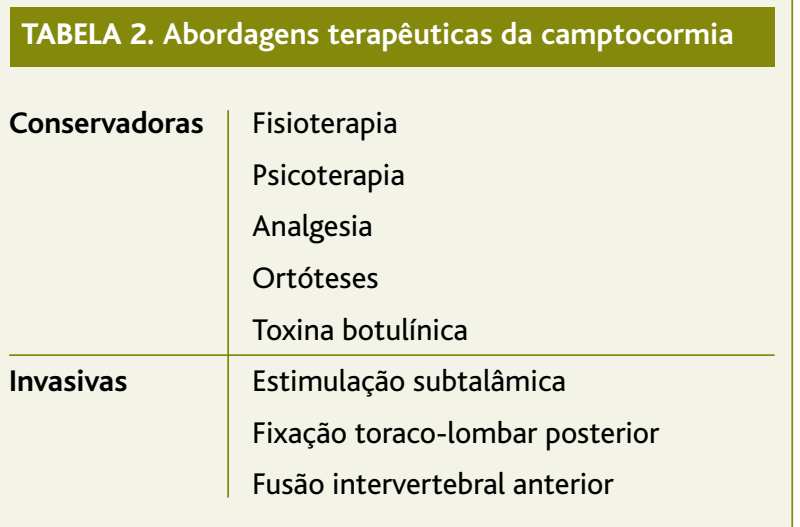


tocormia (efeito-causa e causa-efeito), ${ }_{, 4}^{4}$ como se verifica no caso descrito. A lombalgia é também um sintoma comum. ${ }^{3}$

No caso descrito, o facto de a camptocormia não esbater totalmente, associado à ausência de tremor, discinésia ou outros sintomas extrapiramidais (que possam fazer suspeitar de doença de Parkinson ${ }^{5}$ ou distonia ${ }^{6}$ ), diminui a probabilidade de existir uma doença neurológica de base. Como tal, carece de importância, pelo menos a curto/médio prazo, o seguimento do utente em consulta de neurologia, pelo que a referenciação à consulta desta especialidade poderia não se ter verificado, evitando com isso o tempo de espera e a presença de falsas expectativas de cura da camptocormia sentidas pelo utente.

Após excluir também as causas psiquiátricas ${ }^{7}$ (muito raras) e a toma de fármacos potencialmente causadores de camptocormia, a suspeita diagnóstica mais forte é a de causa ortopédica. Para esta apontam também as alterações documentadas no estudo inicial realizado pelo MF, que referenciou o utente ad initium de forma pertinente e correta à consulta de ortopedia.

As propostas de tratamento da camptocormia podem ser divididas em abordagens conservadoras ou invasivas (Tabela 2). ${ }^{8-9}$

Até ao momento, o utente foi tratado com fisioterapia e hidrocinesioterapia, com melhoria escassa, colocando-se a abordagem de mais opções terapêuticas como uma alternativa pertinente. Destaque para a psicoterapia, muito relevante neste caso clínico, já que o utente se encontra em constante negação quanto ao diagnóstico e suas consequências, buscando ainda uma solução definitiva para o mesmo, solução essa que poderá ser de difícil obtenção. ${ }^{10}$ De realçar ainda a farmacoterapia mais intensiva para o controlo da dor $^{9} \mathrm{e}$ a possibilidade de aplicação de ortótese de alívio toracopélvica anterior, já que estas medidas podem melhorar marcadamente a sua qualidade de vida. ${ }^{9} \mathrm{~A}$ abordagem cirúrgica também deve ser ponderada ${ }^{10}$ para tentativa de melhoria do quadro.

Ainda que as hipóteses de tratamento sejam descritas na literatura como limitadas e pouco consequentes, ${ }^{11}$ a orientação do problema do utente deve ser feita com base numa negociação do plano terapêutico que o envolva globalmente e de forma satisfatória nas tomadas de decisão, procurando assegurar sempre uma relação empática que resulte na adequada gestão das expectativas deste quanto ao plano instituído. Esta abordagem é particularmente importante, já que o utente sente e verbaliza frustração pela demora em conseguir respostas em relação a este problema, que lhe confere diminuição marcada da qualidade de vida.

Conclui-se, portanto, que a abordagem holística ao utente é fundamental para compreender a globalidade do mesmo e dos seus sintomas, de forma a racionalizar o diagnóstico e decidir a forma de referenciação mais adequada, particularmente num diagnóstico tão bizarro como o da camptocormia. A destrinça da possível causa de base é primordial, tendo nisto o MF um papel fundamental, advogando a prestação dos melhores cuidados existentes ao seu utente. A relação cimentada na empatia que o médico ${ }^{11}$ (não só o MF mas também o médico hospitalar) deve procurar ter com o utente é fulcral para o seguimento adequado do mesmo, podendo inclusive ajudar o utente a melhor compreender a relação entre as suas expectativas quanto aos outcomes das intervenções e o descrito na literatura em relação aos seus resultados.

Foi dado pelo utente o consentimento informado, livre e esclarecido para a recolha de dados clínicos, imagens fotográficas e ainda para a publicação deste caso clínico.

\section{AGRADECIMENTOS}

Ao utente, cujo caso foi relatado, pela sua prontidão na recolha dos seus dados, pela disponibilidade para a colheita das imagens fotográficas e ainda pelo consentimento dado para a publicação e divulgação deste caso clínico.

\section{REFERÊNCIAS BIBLIOGRÁFICAS}

1. Kocaaga Z, Bal S, Turan Y, Gurgan A, Esmeli F. Camptocormia and dropped head syndrome as a clinic picture of myotonic myopathy. Joint Bone Spine. 2008;75(6):730-3

2. Finsterer J, StroblW. Presentation, etiology, diagnosis, and management of camptocormia. Eur Neurol. 2010;64(1):1-8

3. Margraf NG, Wrede A, Rohr A, Schulz-Schaeffer WJ, Raethjen J, Eymess A, et al. Camptocormia in idiopathic Parkinson's disease: a focal myopathy of the paravertebral muscles. Mov Disord. 2010;25(5):542-51.

4. Djaldetti R, Melamed E. Camptocormia in Parkinson's disease: new insights. J Neurol Neurosurg Psychiatry. 2006;77(11):1205.

5. Melamed E, Djaldetti R. Camptocormia in Parkinson's disease. J Neurol. 2006;253 Suppl 7:VII14-6.

6. Azher SN, Jankovic J. Camptocormia: pathogenesis, classification, and response to therapy. Neurology. 2005;65(3):355-9.

7. Skidmore F,Anderson K, Fram D, WetherW. Psychogenic camptocormia. Mov Disord. 2007;22(13):1974-5.

8. de Sèze MP, Creuzé A, de Sèze M, Mazaux JM. An orthosis and physio- 
therapy programme for camptocormia: a prospective case study. J Rehabil Med. 2008;40(9):761-5.

9. Peek AC, Quinn N, Casey AT, Etherington G. Thoracolumbar spinal fixation for camptocormia in Parkinson's disease. J Neurol Neurosurg Psychiatry. 2009;80(11):1275-8.

10. Micheli F, Fernández-Pardal MM. DOPA-responsive dystonic camptocormia. Neurology. 2007;68(18):1543.

11. Von Coelln R, Raible A, Gasser T, Asmus F. Ultrasound-guided injection of the iliopsoas muscle with botulinum toxin in camptocormia. Mov Disord. 2008;23(6):889-92.

\section{CONFLITO DE INTERESSES}

Os autores declaram não ter quaisquer conflitos de interesse.

\section{FINANCIAMENTOS}

Os autores declaram que a realização deste caso clínico não foi financiada por nenhuma entidade.

\author{
ENDEREÇO PARA CORRESPONDÊNCIA \\ Jorge Hernâni-Eusébio \\ E-mail: jorgehdossantose@gmail.com \\ https://orcid.org/0000-0002-0372-4798
}

Recebido em 13-08-2019

Aceite para publicação em 12-02-2020

\section{ABSTRACT}

\section{CAMPTOCORMIA: A BIZARRE CASE REPORT AND HOW TO PROPERLY EVALUATE IT}

Introduction: Camptocormia (anterior flexion of the thoracic and lumbar column) is a diagnosis secondary to neurological diseases (mostly Parkinson's disease), orthopedic diseases, and other causes (like pharmacological intoxication and paraneoplastic syndromes). The anterior flexion of the spine due to neurological diseases fully reverses when the patient assumes a supine position. However, that is not observed when camptocormia is due to an orthopedic cause. This finding is particularly important when considering the course of action and proper patient evaluation.

Case report: Male, 65 years old, no relevant medical history or usual medication reported. The patient enters the neurology consultation with marked thoracolumbar anterior flexion. Previously oriented by his general practitioner to an orthopedic appointment, with a CT showing a compression fracture of the T12 vertebrae. The patient underwent physiotherapy and was conducted from the physiatry consultation to neurology with the suspicion of an underlying neurological cause of camptocormia. A physical examination we observed an irreversible camptocormia at supine position and absence of tremor, stiffness, or dyskinesia. Urinary incontinence was ruled out.

Comments: Camptocormia patients might be complex to evaluate. Therefore, the holistic approach is substantial to fully understand the patient's symptoms and the primary cause of camptocormia. In our case report, a non-reversible camptocormia at the supine position, the absence of tremor/extrapyramidal symptoms, and CT findings of an orthopedic cause make the evaluation by neurology less important than the one preferred previously by the general practitioner. We find this approach more convenient in order to manage the patient's expectations regarding the resolution of the problem.

The patient gave his free, clarified, and informed consent regarding the collection of clinical data, photography, and the publication of this case report.

Keywords: Spinal curvatures; Spinal curvatures/diagnosis; Spinal curvatures/etiology; Muscular atrophy/diagnosis; Muscular atrophy/etiology; Camptocormia. 